$\mathrm{DSF}-98 / 6$

INFN-NA-IV-98/6

hep-ph/9803471

\title{
Flavour transitions of Dirac-Majorana neutrinos
}

\author{
Salvatore Esposito 1 and Nicola Tancredi ? \\ Dipartimento di Scienze Fisiche, Università di Napoli "Federico II" \\ Mostra d'Oltremare Pad. 19, I-80125 Napoli Italy \\ and \\ Istituto Nazionale di Fisica Nucleare, Sezione di Napoli \\ Mostra d'Oltremare Pad. 20, I-80125 Napoli Italy
}

\begin{abstract}
From a phenomenological point of view, we study active-active and active-sterile flavour-changing (and flavour-conserving) oscillations of Dirac-Majorana neutrinos both in vacuum and in matter. The general expressions for the transition probabilities in vacuum are reported. We then investigate some interesting consequences following from particular simple forms of the neutrino mass matrices, and for the envisaged scenarios we discuss in detail neutrino propagation in matter. Special emphasis is given to the problem of occurrence of resonant enhancement of active-active and active-sterile neutrino oscillations in a medium. The peculiar novel features related to the Dirac-Majorana nature of neutrinos are particularly pointed out.
\end{abstract}

\footnotetext{
${ }^{1}$ e-mail: sesposito@na.infn.it

${ }^{2}$ e-mail: tancredi@na.infn.it
} 


\section{Introduction}

Today we have several indications in favour of non zero neutrino masses and mixing. The solar neutrino problem, i.e. the observed deficit of solar neutrino fluxes [1], is a well established tool whose resolution requires (almost without doubt [2]) neutrino physics beyond the (minimal) Standard Model. The acceptable solutions to this problem, in terms of vacuum [3] or matter [4] flavour oscillations or spin and flavour oscillations [5] as well as in terms of active-sterile neutrino conversion [6], all need non vanishing neutrino masses and mixing [7, 8, 9, 10].

The second indication in favour of neutrino oscillations come from the observed deficit of atmospheric muon neutrinos with respect to electron neutrinos [1] that can be explained in terms of $\nu_{\mu} \rightarrow \nu_{\tau}$ or $\nu_{\mu} \rightarrow \nu_{e}$ or even active-sterile neutrino transitions [12].

Laboratory direct searches for massive neutrinos only give, at present, upper limits on neutrino masses [13] and the same is valid for reactor and accelerator neutrino oscillations experiments [14, except for the LSND experiment [15] whose results seem to be explained in terms of $\overline{\nu_{\mu}} \rightarrow \overline{\nu_{e}}$ oscillations.

Hints for massive neutrinos also come from cosmology, looking at $\nu_{\tau}$ as the most probable candidate for the hot component of the dark matter [16] and from the observed peculiar velocities of pulsars [17]. On the other hand, in Grand Unified Theories, which attempt to give a unified view of electroweak and strong interactions, massive neutrinos are predicted 18 together with other phenomena violating both lepton numbers and baryon number (such as, for example, proton decay).

However, the most intriguing fact is that a simple scenario with only three massive neutrinos cannot account for the solar neutrino problem, the atmospheric neutrino anomaly and the LSND result. This is because the three squared masses differences $\Delta m^{2}$ for the three oscillation solutions to these problems are all distinct between them: the resonant MSW solution to the solar neutrino problem requires $\Delta m^{2} \sim 10^{-5} \mathrm{eV}^{2}$, while for the atmospheric anomaly $\Delta m^{2} \sim 10^{-2} \mathrm{eV}^{2}$ is needed and $\Delta m^{2} \sim 1 \mathrm{eV}^{2}$ for the LSND result. Many analyses [19] have been conducted for giving a unified view of the three problems in terms of neutrino oscillation (taking into account also the limits from laboratory experiments) and a coherent picture seems to emerge with four massive neutrinos, namely the three known neutrinos plus a sterile neutrino. Note that four neutrino mass eigenstates are needed, but not necessarily more than three neutrino flavour eigenstates. This scenario is easily realized if one considers neutrinos as Dirac-Majorana particles described by the following general mass term in the electroweak lagrangian [10]:

$$
-\mathcal{L}_{m}^{D M}=\sum_{l, l^{\prime}} \bar{\nu}_{l^{\prime} R} M_{l^{\prime} l}^{D} \nu_{l^{\prime} L}+\frac{1}{2} \sum_{l, l^{\prime}} \bar{\nu}_{l^{\prime} R}^{c} M_{l^{\prime} l}^{1} \nu_{l L}+\frac{1}{2} \sum_{l, l^{\prime}} \bar{\nu}_{l^{\prime} L}^{c} M_{l^{\prime} l}^{2} \nu_{l R}+\text { h.c. }
$$

Here $l, l^{\prime}=e, \mu, \tau$ label the three flavour eigenstates and $M_{D}, M_{1}, M_{2}$ are the Dirac and the two Majorana mass matrices which, in general, are hermitian and non diagonal (however, $M_{1}$ and $M_{2}$ have to be symmetric). To construct the mass term in (1) we need the three known left-handed neutrinos (and their antiparticles) and other three right-handed 
sterile neutrinos (and their antiparticles) [1. After the diagonalization of (1) we can obtain in general six mass eigenstates which are Majorana fields; so in this framework we can easily endow the above scenario with four massive neutrinos coming from the experiments. Note that if neutrinos are really described by the mass term in (11), the total lepton number is no longer conserved and peculiar phenomena, as neutrinoless double beta decay and neutrino-antineutrino oscillations can take place.

We stress that (11) is predicted in many GUTs [18] in which the popular "seesaw" mechanism 20] can give rise to very small neutrino masses in a very natural way by supposing $M_{1} \approx 0$ and $M_{D} \ll M_{2}$. However, this is not the only possibility; recently some models assuming $M_{1} \simeq M_{2}$ have been proposed [21] for accounting the three experimental indications on neutrino oscillations discussed above. Here we further explore this last scenario and study flavour transitions of Dirac-Majorana neutrinos from a completely phenomenological point of view, adopting no particular model. This work is a generalization to flavour transitions of previous papers [22, 23] in which we studied peculiar oscillations of Dirac-Majorana neutrinos. We now assume, for simplicity, only two flavours, so $M_{D}$, $M_{1}, M_{2}$ in (1) are $2 \times 2$ matrices in the flavour space. In the following section, the basic vacuum oscillations allowed by (1) are studied and transition probabilities are explicitly given in the general case. Some very interesting consequences due to particularly simple forms of the mass matrix are also investigated. In section 3, given the effective hamiltonian of Dirac-Majorana neutrinos interacting with a medium, resonant matter oscillations are considered along with a qualitative discussion of the phenomenon with the aid of the level crossing diagram. Finally, in section 4, there are our conclusions and remarks.

\section{Dinamical evolution of Dirac-Majorana neutrinos in vacuum}

Let us consider the propagation in vacuum of Dirac-Majorana neutrinos with 4-momentum $k^{\mu}=(\omega, \mathbf{k})$ described by the following lagrangian:

$$
\mathcal{L}=\left(\bar{\nu}, \bar{\nu}^{C}\right)\left(\begin{array}{cc}
\not k & 0 \\
0 & \not k
\end{array}\right)\left(\begin{array}{c}
\nu \\
\nu^{C}
\end{array}\right)-\left(\bar{\nu}, \bar{\nu}^{C}\right)\left(\begin{array}{cc}
M_{D} & M_{M} \\
M_{M} & M_{D}
\end{array}\right)\left(\begin{array}{c}
\nu \\
\nu^{C}
\end{array}\right)
$$

(for convenience we have absorbed a factor $1 / 2$ in $M_{D}$ and $M_{M}$ with respect to the mass terms in (11)). In the chiral Weyl basis for the Dirac gamma matrices, denoting

$$
\nu=\left(\begin{array}{c}
\nu_{L} \\
\nu_{R}
\end{array}\right) \quad \nu^{c}=\left(\begin{array}{c}
\nu_{L}^{c} \\
\nu_{R}^{c}
\end{array}\right)
$$

we have

$$
\left(\begin{array}{cccc}
k & 0 & M_{D} & M_{M} \\
0 & k & M_{M} & M_{D} \\
M_{D} & M_{M} & -k & 0 \\
M_{M} & M_{D} & 0 & -k
\end{array}\right)\left(\begin{array}{c}
\nu_{L}^{c} \\
\nu_{L} \\
\nu_{R}^{c} \\
\nu_{R}
\end{array}\right)=\omega\left(\begin{array}{c}
\nu_{L}^{c} \\
\nu_{L} \\
\nu_{R}^{c} \\
\nu_{R}
\end{array}\right)
$$

\footnotetext{
${ }^{3}$ Obviously, the generalization to more than three families is possible and straightforward
} 
In a more compact form, indicating

$$
n_{L}=\left(\begin{array}{c}
\nu_{L} \\
\nu_{L}^{c}
\end{array}\right) \quad n_{R}=\left(\begin{array}{c}
\nu_{R} \\
\nu_{R}^{c}
\end{array}\right)
$$

and

$$
M=\left(\begin{array}{ll}
M_{D} & M_{M} \\
M_{M} & M_{D}
\end{array}\right)
$$

the equation (41) can be written as

$$
\left(\begin{array}{cc}
k & M \\
M & -k
\end{array}\right)\left(\begin{array}{c}
n_{L} \\
n_{R}
\end{array}\right)=\omega\left(\begin{array}{c}
n_{L} \\
n_{R}
\end{array}\right)
$$

Eqs. (7) shows that, in general, chirality-changing transitions are possible (in fact, in vacuum, chirality is not in general conserved), but these are suppressed with respect to the chirality-preserving ones in the ultrarelativistic limit, because in this limit chirality almost coincides with helicity, which is strictly conserved [23]. Due to this suppression, chirality-changing transitions are not of very practical interest, and we will not consider further these processes. At the leading order in the ultrarelativistic limit $(\omega-k \ll 2 k$, from (7) we deduce that

$$
n_{R} \simeq\left(1-\frac{\omega-k}{2 k}\right) \frac{M}{2 k} n_{L}
$$

while the approximate equation for $n_{L}$ is

$$
H n_{L}=\omega n_{L}
$$

with

$$
H \simeq k+\frac{M^{2}}{2 k}
$$

and the matrix $M$ is given in (6). Here, for simplicity, we limit ourselves to only two flavours, for example $e$ and $\mu$, so that the mass matrices in (6) have in general (assuming $\mathrm{CP}$ conserved) the following non diagonal form:

$$
M_{D}=\left(\begin{array}{cc}
m_{e e}^{D} & m_{e \mu}^{D} \\
m_{e \mu}^{D} & m_{\mu \mu}^{D}
\end{array}\right) \quad M_{M}=\left(\begin{array}{cc}
m_{e e}^{M} & m_{e \mu}^{M} \\
m_{e \mu}^{M} & m_{\mu \mu}^{M}
\end{array}\right)
$$

The $4 \times 4$ hamiltonian in (10) can be easily block-diagonalized by using the unitary matrix

$$
V=\frac{1}{\sqrt{2}}\left(\begin{array}{cc}
I & I \\
-I & I
\end{array}\right)
$$

where $I$ is the $2 \times 2$ identity matrix. In fact, introducing the Majorana states $\widetilde{n_{L}}=V n_{L}$,

$$
\left(\begin{array}{c}
\widetilde{n_{+}} \\
\widetilde{n_{-}}
\end{array}\right)=V\left(\begin{array}{c}
\nu_{L} \\
\nu_{L}^{c}
\end{array}\right)=\frac{1}{\sqrt{2}}\left(\begin{array}{c}
\nu_{L}+\nu_{L}^{c} \\
-\nu_{L}+\nu_{L}^{c}
\end{array}\right)
$$


we can rewrite (9) as

$$
\widetilde{H} \widetilde{n_{L}}=\omega \widetilde{n_{L}}
$$

with

$$
\widetilde{H}=k+\frac{\widetilde{M}^{2}}{2 k}=\left(\begin{array}{cc}
k+\frac{M_{+}^{2}}{2 k} & 0 \\
0 & k+\frac{M_{-}^{2}}{2 k}
\end{array}\right)
$$

where

$$
M_{ \pm}=M_{D} \pm M_{M}
$$

It is now a simple task to completely diagonalize (15) by means of the mixing matrices

$$
U_{ \pm}=\left(\begin{array}{cc}
\cos \theta_{ \pm} & \sin \theta_{ \pm} \\
-\sin \theta_{ \pm} & \cos \theta_{ \pm}
\end{array}\right)
$$

with the mixing angles given by

$$
\tan 2 \theta_{ \pm}=\frac{2 m_{e \mu}^{ \pm}}{m_{e e}^{ \pm}-m_{\mu \mu}^{ \pm}}
$$

$\left(m_{e e}^{ \pm}=m_{e e}^{D} \pm m_{e e}^{M}\right.$ and so on). Indicating with

$$
\begin{aligned}
& m_{1}^{ \pm}=\frac{1}{2}\left(m_{e e}^{ \pm}+m_{\mu \mu}^{ \pm}+\sqrt{\left(m_{e e}^{ \pm}-m_{\mu \mu}^{ \pm}\right)^{2}+4\left(m_{e \mu}^{ \pm}\right)^{2}}\right) \\
& m_{2}^{ \pm}=\frac{1}{2}\left(m_{e e}^{ \pm}+m_{\mu \mu}^{ \pm}-\sqrt{\left(m_{e e}^{ \pm}-m_{\mu \mu}^{ \pm}\right)^{2}+4\left(m_{e \mu}^{ \pm}\right)^{2}}\right)
\end{aligned}
$$

the eigenvalues of the mass matrices in (16), the four energy eigenvalues are

$$
E_{1,2}^{ \pm}=k+\frac{\left(m_{1,2}^{ \pm}\right)^{2}}{2 k}
$$

to which correspond the eigenstates

$$
\left(\begin{array}{c}
\nu_{+} \\
\nu_{-}
\end{array}\right)=U\left(\begin{array}{c}
\widetilde{n_{+}} \\
\widetilde{n_{-}}
\end{array}\right)=\left(\begin{array}{cc}
U_{+} & 0 \\
0 & U_{-}
\end{array}\right)\left(\begin{array}{c}
\widetilde{n_{+}} \\
\widetilde{n_{-}}
\end{array}\right)=U V\left(\begin{array}{c}
\nu_{L} \\
\nu_{L}^{c}
\end{array}\right)
$$

where we have used the shorthand notation

$$
\nu_{ \pm}=\left(\begin{array}{c}
\nu_{1 \pm} \\
\nu_{2 \pm}
\end{array}\right)
$$

Given the relation (22) between the energy eigenstates and the flavour ones, we easily get the time evolution of the states created by weak interactions:

$$
\left(\begin{array}{l}
\mid \nu_{e L}(t)> \\
\mid \nu_{\mu L}(t)> \\
\mid \nu_{e L}^{c}(t)> \\
\mid \nu_{\mu L}^{c}(t)>
\end{array}\right)=V^{T} U^{T} \mathcal{A} U V\left(\begin{array}{l}
\mid \nu_{e L}(0)> \\
\mid \nu_{\mu L}(0)> \\
\mid \nu_{e L}^{c}(0)> \\
\mid \nu_{\mu L}^{c}(0)>
\end{array}\right)
$$


where

$$
\mathcal{A}=\operatorname{diag}\left\{e^{-1 E_{1+} t}, e^{-1 E_{2+} t}, e^{-1 E_{1-} t}, e^{-1 E_{2-} t}\right\}
$$

The transition probabilities $P\left(\nu_{i} \rightarrow \nu_{j}\right)$ are then given by the squared modulus of the corresponding matrix elements $\left|<\nu_{j}(0)\right| \nu_{i}(0)>\left.\right|^{2}$ in (24); after some calculations we obtain

$$
\begin{aligned}
P\left(\nu_{e L} \rightarrow \nu_{\mu L}\right) & =\frac{1}{4}\left(\sin ^{2} 2 \theta_{+} \sin ^{2} \frac{\Delta m_{+}^{2}}{4 k} t+\sin ^{2} 2 \theta_{-} \sin ^{2} \frac{\Delta m_{-}^{2}}{4 k} t\right. \\
& \left.+2 \sin 2 \theta_{+} \sin 2 \theta_{-} \sin \frac{\Delta m_{+}^{2}}{4 k} t \sin \frac{\Delta m_{-}^{2}}{4 k} t \cos \frac{\Sigma}{4 k} t\right) \\
P\left(\nu_{e L} \rightarrow \nu_{\mu L}^{c}\right)= & \frac{1}{4}\left(\sin ^{2} 2 \theta_{+} \sin ^{2} \frac{\Delta m_{+}^{2}}{4 k} t+\sin ^{2} 2 \theta_{-} \sin ^{2} \frac{\Delta m_{-}^{2}}{4 k} t\right. \\
P\left(\nu_{e L} \rightarrow \nu_{e L}^{c}\right)= & \left.2 \sin 2 \theta_{+} \sin 2 \theta_{-} \sin \frac{\Delta m_{+}^{2}}{4 k} t \sin \frac{\Delta m_{-}^{2}}{4 k} t \cos \frac{\Sigma}{4 k} t\right) \\
& +c_{+}^{2} c_{-}^{2} \sin ^{2} \frac{\Sigma-\Delta m_{+}^{2}+\Delta m_{-}^{2}}{8 k} t+c_{+}^{2} s_{-}^{2} \sin ^{2} \frac{\Sigma-\Delta m_{+}^{2}-\Delta m_{-}^{2}}{8 k} t \\
& -c_{+}^{2} s_{+}^{2} \sin ^{2} \frac{\Delta m_{+}^{2}}{4 k} t-c_{-}^{2} s_{-}^{2} \sin ^{2} \frac{\Delta m_{-}^{2}}{4 k} t
\end{aligned}
$$

and for the survival probability

$$
\begin{aligned}
P\left(\nu_{e L} \rightarrow \nu_{e L}\right)=1 & -c_{+}^{2} c_{-}^{2} \sin ^{2} \frac{\Sigma-\Delta m_{+}^{2}+\Delta m_{-}^{2}}{8 k} t-c_{+}^{2} s_{-}^{2} \sin ^{2} \frac{\Sigma-\Delta m_{+}^{2}-\Delta m_{-}^{2}}{8 k} t \\
& -s_{+}^{2} c_{-}^{2} \sin ^{2} \frac{\Sigma+\Delta m_{+}^{2}+\Delta m_{-}^{2}}{8 k} t-s_{+}^{2} s_{-}^{2} \sin ^{2} \frac{\Sigma+\Delta m_{+}^{2}-\Delta m_{-}^{2}}{8 k} t \\
& -c_{+}^{2} s_{+}^{2} \sin ^{2} \frac{\Delta m_{+}^{2}}{4 k} t-c_{-}^{2} s_{-}^{2} \sin ^{2} \frac{\Delta m_{-}^{2}}{4 k} t
\end{aligned}
$$

(we have adopted the shorthand notation $c_{ \pm}=\cos \theta_{ \pm}, s_{ \pm}=\sin \theta_{ \pm}$). These probabilities in general depend on 5 parameters of the underlying theory, that is 2 mixing angles $\theta_{ \pm}$, and 3 mass parameters $\Delta m_{ \pm}^{2}=m_{2 \pm}^{2}-m_{2 \pm}^{1}, \Sigma=m_{1+}^{2}+m_{2+}^{2}-m_{1-}^{2}-m_{2-}^{2}$.

Note that in the limit of zero mixing angles all transition probabilities vanish except $P\left(\nu_{e L} \rightarrow \nu_{e L}^{c}\right)$ for which we recover the Pontecorvo oscillation formula [24, 22]: in this limit only neutrino-antineutrino oscillations with no flavour change are possible with both Dirac and Majorana mass terms.

The obtained results for the transition probabilities in the general case, eqs. (17)-(19), are rather complicate and then difficult to analyze. In the following we discuss some very interesting particular cases obtained for peculiar forms of Dirac and Majorana mass matrices. 


\subsection{Pure Dirac and pure Majorana neutrinos.}

For the two limiting cases

$$
M_{D} \neq 0 \quad M_{M}=0
$$

(pure Dirac neutrinos) and

$$
M_{D}=0 \quad M_{M} \neq 0
$$

(pure Majorana neutrinos) we have

$$
\theta_{+}=\theta_{-} \quad M_{+}^{2}=M_{-}^{2}
$$

so that

$$
\begin{aligned}
P\left(\nu_{e L} \rightarrow \nu_{\mu L}\right) & =\sin ^{2} 2 \theta \sin ^{2} \frac{\Delta m^{2}}{4 k} t \\
P\left(\nu_{e L} \rightarrow \nu_{\mu L}^{c}\right) & =0 \\
P\left(\nu_{e L} \rightarrow \nu_{e L}^{c}\right) & =0
\end{aligned}
$$

As we know, in these frameworks we have no neutrino-antineutrino oscillations but only flavour transitions, for which we recover the standard results [0]. Note that for pure Dirac and pure Majorana neutrinos the result is the same, but this holds only in the ultrarelativistic limit [25].

\subsection{The cases of Dirac mixing and Majorana masses and vice- versa.}

In general, all the elements of the $M_{D}$ and $M_{M}$ mass matrices in (2) are non zero; however, we can constrain these by making some physical ansatz.

Looking at the quark sector of the Standard Model we note that quarks are Dirac particles (obviously!) and flavour (weak interacting) eigenstates are mixed to give the mass eigenstates. In analogy, we can assume that also for neutrinos the Dirac mass matrix is responsible for the mixing of the flavour eigenstates. Nevertheless, we know that neutrinos, if massive, are much more light than the corresponding quarks (see the limits reported in [13]) so that one can think that their masses (but not necessarily mixings) are generated by a Majorana mass term. The most simple forms for $M_{D}$ and $M_{M}$ translating these two ansatz are then

$$
M_{D}=\left(\begin{array}{cc}
0 & m_{e \mu}^{D} \\
m_{e \mu}^{D} & 0
\end{array}\right) \quad M_{M}=\left(\begin{array}{cc}
m_{e e}^{M} & 0 \\
0 & m_{\mu \mu}^{M}
\end{array}\right)
$$

We now explore the implications of (36). The first one is that

$$
U_{-}=U_{+}^{\dagger}
$$




$$
M_{+}^{2}=M_{-}^{2}
$$

i.e. + states and - states have equal mass eigenvalues but + states are mixed between them in a way just opposite to that of - states. As a consequence

$$
\begin{gathered}
\theta_{-}=-\theta_{+} \\
\Delta m_{+}^{2}=\Delta m_{-}^{2} \\
\Sigma=0
\end{gathered}
$$

Inserting these in the expressions for the transition probabilities (17)-(19) we get

$$
\begin{aligned}
& P\left(\nu_{e L} \rightarrow \nu_{\mu L}\right)=0 \\
& P\left(\nu_{e L} \rightarrow \nu_{\mu L}^{c}\right)=\sin ^{2} 2 \theta_{+} \sin ^{2} \frac{\Delta m_{+}^{2}}{4 k} t \\
& P\left(\nu_{e L} \rightarrow \nu_{e L}^{c}\right)=0
\end{aligned}
$$

We see that, in this case, pure flavour oscillations and pure neutrino-antineutrino (Pontecorvo) oscillations are not allowed, while only flavour changing neutrino-antineutrino transitions are predicted. It is interesting to note that for the latter, the expression for the transition probability has the same form as (33). As we will remark below, this has implications on the interpretation of disappearance neutrino oscillation experiments.

On the contrary to the ansatz just analyzed, we can further explore the possibility that neutrino mixing is given only by the Majorana mass term, while masses are generated directly by the Dirac term. We then consider the following mass matrices:

$$
M_{D}=\left(\begin{array}{cc}
m_{e e}^{D} & 0 \\
0 & m_{\mu \mu}^{D}
\end{array}\right) \quad M_{M}=\left(\begin{array}{cc}
0 & m_{e \mu}^{M} \\
m_{e \mu}^{M} & 0
\end{array}\right)
$$

Also in this case we find that relations (37),(38) hold, so that again we have eqs. (42)(44) for the transition probabilities. The fact that both cases analyzed in this paragraph lead to the same phenomenological predictions is analogous to that encountered in the previous paragraph, where (30) and (31) also gave identical results. We then observe a symmetry between Dirac and Majorana mass terms.

\subsection{The case of degenerate Dirac and Majorana mixing}

Another interesting ansatz is to suppose that neutrino mixing is generated by Dirac and

Majorana mass terms with the same strength. This can be simply implemented by using

$$
M_{D}=\left(\begin{array}{cc}
m_{e e}^{D} & m_{e \mu} \\
m_{e \mu} & m_{\mu \mu}^{D}
\end{array}\right) \quad M_{M}=\left(\begin{array}{cc}
0 & m_{e \mu} \\
m_{e \mu} & 0
\end{array}\right)
$$

or

$$
M_{D}=\left(\begin{array}{cc}
0 & m_{e \mu} \\
m_{e \mu} & 0
\end{array}\right) \quad M_{M}=\left(\begin{array}{cc}
m_{e e}^{M} & m_{e \mu} \\
m_{e \mu} & m_{\mu \mu}^{M}
\end{array}\right)
$$


In both cases we have one non-vanishing mixing angle and 2 (nearly) independent mass parameter; more precisely

$$
\begin{gathered}
\theta_{-}=0 \\
\Delta m_{-}^{2}=\cos 2 \theta_{+} \Delta m_{+}^{2}
\end{gathered}
$$

while $\Sigma$ is given by

$$
\Sigma=\frac{2 \sin ^{2} 2 \theta_{+}\left(\Delta m_{+}^{2}\right)^{2}}{\left(m_{1-}+m_{2-}\right)^{2}}
$$

Note that in the limit of zero mixing angle we are left with only one mass parameter $(\Sigma=0)$. Instead for arbitrary mixing, in the present case, if the states $\nu_{1+}, \nu_{2+}$ are degenerate in mass $\left(\Delta m_{+}^{2}=0\right)$ then all the four states $\nu_{1 \pm}, \nu_{2 \pm}$ are degenerate $\left(\Delta m_{+}^{2}=\right.$ $\left.\Delta m_{-}^{2}=\Sigma=0\right)$ so that no transition can occur.

Inserting (48), (49) in (17)-(19) we now observe that all the transition probabilities are different from zero, and are given by

$$
\begin{aligned}
& P\left(\nu_{e L} \rightarrow \nu_{\mu L}\right)=P\left(\nu_{e L} \rightarrow \nu_{\mu L}^{c}\right)=\frac{1}{4} \sin ^{2} 2 \theta_{+} \sin ^{2} \frac{\Delta m_{+}^{2}}{4 k} t \\
& P\left(\nu_{e L} \rightarrow \nu_{e L}^{c}\right)=c_{+}^{2} \sin ^{2}\left(\frac{\Sigma-2 s_{+} \Delta m_{+}^{2}}{8 k} t\right)+s_{+}^{2} \sin ^{2}\left(\frac{\Sigma+2 c_{+} \Delta m_{+}^{2}}{8 k} t\right) \\
&-c_{+}^{2} s_{+}^{2} \sin ^{2}\left(\frac{\Delta m_{+}^{2}}{4 k} t\right)
\end{aligned}
$$

Interestingly, let us note that both $\nu_{e L} \rightarrow \nu_{\mu L}$ and $\nu_{e L} \rightarrow \nu_{\mu L}^{c}$ transitions have the same probability, whose form is identical to (33) and (43) apart a constant suppression factor for the oscillation amplitude of $1 / 4$. For degenerate Dirac-Majorana mixing we then predict non vanishing flavour-conserving oscillations and equal probabilities for flavour-changing ones.

\section{Dirac-Majorana neutrino oscillations in matter}

In this section we generalize the MSW theory [8] to take into account the Dirac-Majorana nature of neutrinos.

Let us consider neutrinos travelling in a medium with constant density, whose electron and neutron number densities are given by $N_{e}$ and $N_{n}$, respectively. In the flavour basis, the evolution equations are simply given by (4) where the energy $\omega$ is substituted by $\omega-V, V$ being the effective potential experienced by a given neutrino state in the medium, namely 8, 26, 25]

$$
\begin{aligned}
& V_{\nu_{e L}}=-V_{\nu_{e R}^{c}}=\sqrt{2} G_{F}\left(N_{e}-\frac{1}{2} N_{n}\right) \\
& V_{\nu_{\mu L}}=-V_{\nu_{\mu R}^{c}}=-\frac{G_{F}}{\sqrt{2}} N_{n} \\
& V=0 \quad \text { for all the other sterile states }
\end{aligned}
$$


Here $G_{F}$ is the Fermi coupling constant, and for simplicity we consider only non magnetized media (the generalization to these media is straightforward following [26]). In compact form, the evolution equations are then given by

$$
\left(\begin{array}{cc}
k+V_{L} & M \\
M & -k-V_{R}
\end{array}\right)\left(\begin{array}{c}
n_{L} \\
n_{R}
\end{array}\right)=\omega\left(\begin{array}{c}
n_{L} \\
n_{R}
\end{array}\right)
$$

where

$$
\begin{aligned}
V_{L} & =\operatorname{diag}\left\{V_{\nu_{e L}}, V_{\nu_{\mu L}}, 0,0\right\} \\
V_{R} & =\operatorname{diag}\left\{0,0,-V_{\nu_{e R}^{c}},-V_{\nu_{\mu R}^{c}}\right\}
\end{aligned}
$$

In the ultrarelativistic limit, and for $V_{\nu_{e L}}, V_{\nu_{\mu L}} \ll k$, we can again separate the evolution of $n_{L}$ and $n_{R}$ states, and in particular we get

$$
H_{m} n_{L}=\omega n_{L}
$$

with the effective hamiltonian given by

$$
H_{m} \simeq k+\frac{M^{2}}{2 k}+V_{L}
$$

In terms of the physical mass and mixing parameters, subtracting from $H_{m}$ terms proportional to the identity matrix which contribute with an irrelevant common phase factor to the wave functions, the effective hamiltonian in the flavour basis can be explicitly written as

$$
H_{m}=\frac{1}{4}\left(\begin{array}{cccc}
-D_{+c}+4 V_{\nu_{e L}} & -D_{+s} & \sigma-D_{-c} & -D_{-s} \\
-D_{+s} & D_{+c}+4 V_{\nu_{\mu L}} & -D_{-s} & \sigma+D_{-c} \\
\sigma-D_{-c} & -D_{-s} & -D_{+c} & -D_{+s} \\
-D_{-s} & \sigma+D_{-c} & -D_{+s} & D_{+c}
\end{array}\right)
$$

where we have used the notations

$$
\begin{aligned}
D_{ \pm c} & \simeq \frac{\Delta m_{+}^{2}}{2 k} c_{2+} \pm \frac{\Delta m_{-}^{2}}{2 k} c_{2-} \\
D_{ \pm s} & \simeq \frac{\Delta m_{+}^{2}}{2 k} s_{2+} \pm \frac{\Delta m_{-}^{2}}{2 k} s_{2-} \\
\sigma & =\frac{\Sigma}{2 k}
\end{aligned}
$$

Diagonalizing $H_{m}$ in (62) we then get the matter mass eigenstates from which the transition probabilities in matter can be obtained. Before dealing with this point, we first want to discuss the occurrence of resonances in neutrino matter oscillations. 


\subsection{Resonance conditions}

Resonant enhancement of oscillations takes place when two unperturbed (mixing equals zero) energy levels cross between them [8]. The resonance conditions can then be (approximately) obtained by equating the diagonal elements of $H_{m}$ in (62).

For resonant $\nu_{e L} \rightarrow \nu_{\mu L}$ transitions we have the following condition

$$
\frac{\Delta m_{+}^{2}}{2 k} \cos 2 \theta_{+}+\frac{\Delta m_{-}^{2}}{2 k} \cos 2 \theta_{-}=2 \sqrt{2} G_{F} N_{e}
$$

while for $\nu_{e L} \rightarrow \nu_{\mu L}^{c}$ and $\nu_{\mu L} \rightarrow \nu_{e L}^{c}$

$$
\begin{aligned}
\frac{\Delta m_{+}^{2}}{2 k} \cos 2 \theta_{+}+\frac{\Delta m_{-}^{2}}{2 k} \cos 2 \theta_{-} & =2 \sqrt{2} G_{F}\left(N_{e}-\frac{1}{2} N_{n}\right) \\
\frac{\Delta m_{+}^{2}}{2 k} \cos 2 \theta_{+}+\frac{\Delta m_{-}^{2}}{2 k} \cos 2 \theta_{-} & =\sqrt{2} G_{F} N_{n}
\end{aligned}
$$

respectively. Instead, as already found in [23], the active-sterile flavour-conserving matter transitions $\nu_{e L} \rightarrow \nu_{e L}^{c}$ and $\nu_{\mu L} \rightarrow \nu_{\mu L}^{c}$ have a maximum amplitude only if

$$
V_{\nu_{e}}=0
$$

or

$$
V_{\nu_{\mu}}=0
$$

respectively 1 .

The relation (66) generalizes to Dirac-Majorana neutrinos the resonance condition for flavour oscillations obtained in [\&] for pure Dirac or pure Majorana (ultrarelativistic) neutrinos:

$$
\frac{\Delta m^{2}}{2 k} \cos 2 \theta=\sqrt{2} G_{F} N_{e}
$$

An important novel feature emerging from (66) is that, on the contrary to what happens for pure Dirac or pure Majorana neutrinos (eq. (71)), the resonance condition is ruled by two squared masses differences so that, even if two or three of four mass eigenvalues are degenerate, the enhancement of oscillations can still take place (note that the resonance density in (66) is shifted with respect to that occurring for (71) towards lower values). The same is valid for active-sterile (flavour-changing) transitions. Note, however, that the resonance conditions do not depend on the $\Sigma$ parameter.

For the particular case in which the Dirac and Majorana mass matrices are given by (36) or (45), only one transition $\left(\nu_{e L} \rightarrow \nu_{\mu L}^{c}\right)$ can occur, and for this the resonance condition reduces to

$$
\frac{\Delta m_{+}^{2}}{2 k} \cos 2 \theta_{+}=\sqrt{2} G_{F}\left(N_{e}-\frac{1}{2} N_{n}\right)
$$

\footnotetext{
${ }^{4}$ Note that, while eq. $(70)$ is realized only in vacuum, relation $(\sqrt[69]{6)}$ can be satisfied also in a medium with $N_{e}=N_{n} / 2$. Such a condition can be achieved in the first stages of the neutronization phase of a neutron star.
} 
Instead, for the mass matrices taking the form in (46) or (47) all the transition discussed in the general case can be resonant, but now they are all ruled by only one squared masses difference. So, the resonance condition for $\nu_{e L} \rightarrow \nu_{\mu L}$

$$
\frac{\Delta m_{+}^{2}}{2 k} \cos 2 \theta_{+}=\sqrt{2} G_{F} N_{e}
$$

is phenomenologically equivalent to that for pure Dirac or pure Majorana neutrinos, while the one for $\nu_{e L} \rightarrow \nu_{\mu L}^{c}$ is again given by (72).

After this qualitative discussion on the resonant enhancement of matter oscillations, we now proceed to find the expressions for the transition probabilities. As for the vacuum, this can be achieved also in the general case (described by the hamiltonian $H_{m}$ in (62)) because the eigenvalue equation corresponds to a fourth degree algebraic equation whose solutions are known analytically. However, the resulting expressions for the probabilities are very involved and not very informative as (and much more than) in the vacuum case. So we will only consider the particular scenarios envisaged in the previous section and for these we will single out the explicit form of the transition probabilities.

\subsection{Pure Dirac and pure Majorana neutrinos}

Given (30) or (31) the problem is separable into the diagonalization of two $2 \times 2$ effective hamiltonians, one for the active left-handed neutrino states and the other for the sterile left-handed antineutrino states (which propagate freely as in vacuum) for the Dirac case or for the active right-handed antineutrino states for the Majorana case. In both cases we have

$$
H_{m} \nu_{L}=\omega \nu_{L}
$$

with

$$
H_{m} \simeq k+\frac{M_{D, M}^{2}}{2 k}+\left(\begin{array}{cc}
V_{\nu_{e L}} & 0 \\
0 & V_{\nu_{\mu L}}
\end{array}\right)
$$

and so we recover the standard MSW theory [B].

No active-sterile (flavour-changing) transition occurs in this case, as in vacuum.

\subsection{The cases of Dirac mixing and Majorana masses and vice- versa}

Let us now assume that mass matrices are given by (30) or (45) and substitute relations (39)-(41) in the effective hamiltonian (62). Inspired from the results obtained for the vacuum case, it is useful to introduce the permutated flavour basis

$$
\left(\begin{array}{c}
\nu_{e L} \\
\nu_{\mu L}^{c} \\
\nu_{e L}^{c} \\
\nu_{\mu L}
\end{array}\right)
$$


In this basis, the problem is again separable because the effective hamiltonian becomes block-diagonal. We then have

$$
\begin{aligned}
& H_{m}^{(1)}\left(\begin{array}{c}
\nu_{e L} \\
\nu_{\mu L}^{c}
\end{array}\right)=\omega\left(\begin{array}{c}
\nu_{e L} \\
\nu_{\mu L}^{c}
\end{array}\right) \\
& H_{m}^{(2)}\left(\begin{array}{c}
\nu_{e L}^{c} \\
\nu_{\mu L}
\end{array}\right)=\omega\left(\begin{array}{c}
\nu_{e L}^{c} \\
\nu_{\mu L}
\end{array}\right)
\end{aligned}
$$

with

$$
\begin{aligned}
& H_{m}^{(1)} \simeq k+\frac{\mathcal{M}^{\epsilon}}{2 k}+\left(\begin{array}{cc}
V_{\nu_{e L}} & 0 \\
0 & 0
\end{array}\right) \\
& H_{m}^{(2)} \simeq k+\frac{\mathcal{M}^{\epsilon}}{2 k}+\left(\begin{array}{cc}
0 & 0 \\
0 & V_{\nu_{\mu L}}
\end{array}\right)
\end{aligned}
$$

where the mass matrix $\mathcal{M}$ is

$$
\mathcal{M}=\left(\begin{array}{ll}
m_{e e}^{D} & m_{e \mu}^{M} \\
m_{e \mu}^{M} & m_{\mu \mu}^{D}
\end{array}\right)
$$

Let us focus, for example, on (79). It has the same form of the $2 \times 2$ effective $e-\mu \mathrm{MSW}$ hamiltonian (75); the expressions for the transition probabilities in matter are then

$$
\begin{aligned}
& P\left(\nu_{e L} \rightarrow \nu_{\mu L}\right)=0 \\
& P\left(\nu_{e L} \rightarrow \nu_{\mu L}^{c}\right)=\sin ^{2} 2 \theta_{+}^{m} \sin ^{2} \frac{\pi x}{L_{m}} \\
& P\left(\nu_{e L} \rightarrow \nu_{e L}^{c}\right)=0
\end{aligned}
$$

$(x \simeq t)$ where the effective mixing angle in matter is given by

$$
\sin 2 \theta_{+}^{m}=\frac{\frac{\Delta m_{+}^{2}}{2 k} \sin 2 \theta_{+}}{\sqrt{\left(\frac{\Delta m_{+}^{2}}{2 k} \cos 2 \theta_{+}-\sqrt{2} G_{F}\left(N_{e}-\frac{1}{2} N_{n}\right)\right)^{2}+\left(\frac{\Delta m_{+}^{2}}{2 k} \sin 2 \theta_{+}\right)^{2}}}
$$

while the effective oscillation length by

$$
L_{m}=\frac{2 \pi}{\sqrt{\left(\frac{\Delta m_{+}^{2}}{2 k} \cos 2 \theta_{+}-\sqrt{2} G_{F}\left(N_{e}-\frac{1}{2} N_{n}\right)\right)^{2}+\left(\frac{\Delta m_{+}^{2}}{2 k} \sin 2 \theta_{+}\right)^{2}}}
$$

From (85) we then obtain again that the transitions $\nu_{e L} \rightarrow \nu_{\mu L}^{c}$ are resonantly amplified if the resonance condition $(\sqrt{72})$ is fulfilled.

At this point we stress the fact that for Dirac-Majorana neutrinos described by the mass matrices in (30) or (45) the MSW theory [8] applies practically unmodified to $\nu_{e L} \rightarrow \nu_{\mu L}^{c}$ instead of $\nu_{e L} \rightarrow \nu_{\mu L}$ flavour transitions, and so the phenomenological implications for disappearance experiments are the same for the two cases. In particular, the analysis performed in [6] for the solar neutrino problem holds true for the present case, and from that the values of $\Delta m_{+}^{2}$ and $\sin ^{2} 2 \theta_{+}$able to solve the puzzle can be extracted. 


\subsection{The case of degenerate Dirac-Majorana mixing}

Let us now turn on the last model considered in the previous section, with the mass matrices given by (46) or (47). From (48), (49) we then find that the proper effective hamiltonian to diagonalize is

$$
\begin{aligned}
H_{m} & =\frac{1}{8 k}\left(\begin{array}{cccc}
-2 \Delta m_{+}^{2} \cos 2 \theta_{+} & -\Delta m_{+}^{2} \sin 2 \theta_{+} & \Sigma & -\Delta m_{+}^{2} \sin 2 \theta_{+} \\
-\Delta m_{+}^{2} \sin 2 \theta_{+} & 2 \Delta m_{+}^{2} \cos 2 \theta_{+} & -\Delta m_{+}^{2} \sin 2 \theta_{+} & \Sigma \\
\Sigma & -\Delta m_{+}^{2} \sin 2 \theta_{+} & -2 \Delta m_{+}^{2} \cos 2 \theta_{+} & -\Delta m_{+}^{2} \sin 2 \theta_{+} \\
-\Delta m_{+}^{2} \sin 2 \theta_{+} & \Sigma & -\Delta m_{+}^{2} \sin 2 \theta_{+} & 2 \Delta m_{+}^{2} \cos 2 \theta_{+}
\end{array}\right)+ \\
& +\operatorname{diag}\left\{V_{\nu_{e L}}, V_{\nu_{\mu L}}, 0,0\right\}
\end{aligned}
$$

Because of the presence of $V_{\nu_{\mu L}}$, we observe that the submatrices corresponding to the subsystem $\left(\nu_{e L}, \nu_{\mu L}\right)$ and $\left(\nu_{e L}, \nu_{\mu L}^{c}\right)$ are now not equal (as instead happened for the vacuum case) so that $\nu_{e L} \rightarrow \nu_{\mu L}$ and $\nu_{e L} \rightarrow \nu_{\mu L}^{c}$ oscillations have different transition probabilities. The interactions with the medium remove this sort of degeneracy; this was manifest already in the expressions for the resonance conditions (66) and (67).

The exact eigenvalue problem for $H_{m}$ in (87) involves again complicated solutions of a fourth-degree equation. Instead of giving the general expressions for the transition probabilities, it is more useful to discuss qualitatively the oscillations pattern which is very similar to the general one described by $H_{m}$ in (62) since in both cases all the transition between the different flavour states can take place (the main differences between the two cases will be remarked in the following section).

For a medium with varying density we can qualitatively analyze neutrino propagation in it with the help of a level crossing diagram, depicted (for the present case) in Fig. 1. Full lines represent the eigenvalues of $H_{m}$ in (87) plotted against the medium density, while the dashed lines correspond to the unperturbed (no mixing) energy eigenvalues (i.e. to the diagonal elements of $H_{m}$ with $\theta_{+}=0$, since the non diagonal elements all vanish in the zero mixing limit). The crossing points of the unperturbed levels (approximatively) identify the resonance densities $\rho_{R i}$ for the transitions $\nu_{e L} \rightarrow \nu_{\mu L}(\mathrm{R} 1), \nu_{\mu L} \rightarrow \nu_{e L}^{c}$ (R2) and $\nu_{e L} \rightarrow \nu_{\mu L}^{c}$ (R3). The relative positions of the three resonance points depend on the $Y_{e}=Z / A$ ratio of the considered medium; as one can see from (66)-(68),

$$
\begin{aligned}
& \rho_{R 1} \sim Y_{e}^{-1} \\
& \rho_{R 2} \sim\left|\frac{1-Y_{e}}{2}\right|^{-1} \\
& \rho_{R 3} \sim\left|\frac{3 Y_{e}-1}{2}\right|^{-1}
\end{aligned}
$$

In Fig. 1 we have chosen $Y_{e}=0.48$; in the particularly common case in which $Y_{e}=0.5$ we would have that both R2 and R3 occur at the same density.

Let us now focus on the evolution od Dirac-Majorana neutrinos propagating in a varying density medium, such as for example the Sun or another star like this. Neutrinos are produced deep in the star, at high densities, in flavour eigenstates (typically $\nu_{e L}$ ) and then 
move out towards low density regions. As can be seen from Fig. 1, in fact, at high densities mixing effects can be neglected and the energy eigenvalues practically coincide with the unperturbed ones. The subsequent evolution, with decreasing density, depends whether the resonances are crossed adiabatically or not (i.e., qualitatively, if neutrinos travelling in the medium "see" a density which varies very slowly during their path or not). If the adiabaticity condition [27] is fulfilled (at each resonance), neutrinos evolve according to the unbroken lines; otherwise at a given resonance there is a non vanishing probability for "jumping" from one level to another one [27]. So, following Fig. 1, if for example we have a $\nu_{e L} \simeq \nu_{4}$ at high densities and the resonance R3 is crossed adiabatically, at intermediate densities we again encounter a $\nu_{4}$ but now $\nu_{4} \simeq \nu_{\mu L}^{c}$ : we have had a transformation of an active $\nu_{e L}$ into a sterile $\nu_{\mu L}^{c}$. Instead, if R3 is crossed non adiabatically so that there is a non zero probability to jump onto the $\nu_{1}$ state, at intermediate densities we encounter (with a certain probability) a $\nu_{1} \simeq \nu_{e L}$ and practically we have had no conversion. But moving towards lower density another resonance point (R1) is present: if this is crossed adiabatically, then after that we have a $\nu_{1} \simeq \nu_{\mu L}$ (active-active $\nu_{e L} \rightarrow \nu_{\mu L}$ conversion), otherwise we have a jump onto the $\nu_{2}$ state which (after the resonance) is approximatively a $\nu_{e L}$ (no conversion).

Confronting the present scenario with that of the usual MSW theory, we see that now efficient active-active flavour-changing conversions can be obtained only if the resonance for active-sterile transitions ( $\mathrm{R} 3$ or R2) is crossed non adiabatically while that for activeactive transition (R1) is crossed adiabatically. But the important novel feature regarding Dirac-Majorana neutrinos is that exiting from the medium we do not have pure flavour states. In fact, as one can see from Fig. 1 and contrarily to what happens for the standard MSW theory, at very low density (approaching the vacuum) the energy eigenstates (solid lines) do not approach flavour eigenstates (dashed lines). This is a genuine feature of the Dirac-Majorana nature of neutrinos and it can be shown that (in the present case) this is due to the fact that in the limit of zero mixing the $\Sigma$ parameter vanishes (see eq. (50)) 0 . However, this is not a completely surprising feature, because we already know that in vacuum even for zero mixing the pure flavour eigenstates are not the physical eigenstates, which are instead given by the Majorana combinations $\widetilde{n_{ \pm}}$in (13). The level crossing diagram in Fig. 1 for zero density is just an expression of this physical fact. For zero mixing we have only two (doubly degenerate) energy eigenvalues $\pm \frac{\Delta m_{+}^{2}}{4 k}$ while, switching on the mixing, four non degenerate energy eigenvalues appear,

$$
\begin{gathered}
\frac{1}{8 k}\left( \pm 2 \Delta m_{+}^{2}+\Sigma\right) \\
\frac{1}{8 k}\left( \pm 2 \Delta m_{+}^{2} \cos 2 \theta_{+}-\Sigma\right)
\end{gathered}
$$

and the physical eigenstates at the exit of the medium are just $\nu_{1}, \nu_{2}, \nu_{3}, \nu_{4}=\widetilde{n_{ \pm}}$ corresponding to the energy values given in (91), (92).

\footnotetext{
${ }^{5}$ In fact, keeping $\Sigma$ fixed in the limit $\theta_{+} \rightarrow 0$, at very low density the dashed lines approach the solid ones
} 


\section{Concluding remarks}

In this paper we have studied the propagation both in vacuum and in matter of DiracMajorana neutrinos and analyzed active-active (flavour-changing) as well as active-sterile transitions, which are, in general, both possible.

For vacuum oscillations, in section 2 we have given the general expressions for the transitions probabilities for $\nu_{e L} \rightarrow \nu_{\mu L}, \nu_{e L} \rightarrow \nu_{\mu L}^{c}, \nu_{e L} \rightarrow \nu_{e L}^{c}$ We have then discussed some interesting limiting cases for Dirac $\left(M_{D}\right)$ and Majorana $\left(M_{M}\right)$ mass matrices. For pure Dirac $\left(M_{M}=0\right)$ or pure Majorana $\left(M_{D}=0\right)$ neutrinos obviously we recover the usual flavour oscillation formulae [7], while for both $M_{D}$ and $M_{M}$ non vanishing and diagonal the Pontecorvo formula [24] for neutrino-antineutrino (active-sterile) oscillations is obtained [22].

An interesting non trivial case is that with $M_{D}$ and $M_{M}$ given by (36) or (45) which implement the idea that neutrino mixing is essentially ruled only by the Dirac mass term while the Majorana mass term is diagonal or vice-versa, respectively. In both cases, neither pure flavour oscillations nor Pontecorvo oscillations are predicted, but only flavour-changing active-sterile transitions, such as $\nu_{e L} \rightarrow \nu_{\mu L}^{c}$, are possible. Remarkably, the transition probability for these is identical in form to that for flavour oscillations for pure Dirac or Majorana neutrinos, and this holds both in vacuum and in matter. For the latter, the resonance condition is only shifted by the neutral current contribution of $\nu_{e L}$ to the effective potential. So, for example, the solution to the solar neutrino problem in terms of active-sterile neutrino oscillations proposed in [6] applies unmodified to the present scheme.

Another interesting case, even if a bit more complicate, has been analysed for the mass matrices in (46) or (47), which implements the idea that neutrino mixing is given by the Dirac and Majorana mass terms with the same strength. In this case, $\nu_{e L} \rightarrow \nu_{\mu L}$, $\nu_{e L} \rightarrow \nu_{\mu L}^{c}, \nu_{e L} \rightarrow \nu_{e L}^{c}$ transitions are all possible and, in vacuum, the first two have the same transition probability, which is also identical in form to that obtained in the previous case, except for a constant suppression factor in the amplitude of oscillations of $1 / 4$. Also in matter the pattern of neutrino transitions present in the general case is (qualitatively) reproduced in this peculiar scheme. In particular, all the flavour changing oscillations can be resonantly amplified while Pontecorvo $\nu_{e L} \rightarrow \nu_{e L}^{c}$ matter oscillations have maximum amplitude only for a given electron to neutron number ratio (see eq. (69) and the related footnote); the resonance conditions were discussed in section 3.1 .

Given the multiresonance structure of the oscillations pattern, it is then interesting to follow the evolution of a $\nu_{e L}$, for example, in a varying density medium such as the Sun; this has been done in section 3.4 with the help of a level crossing diagram reported in Fig. 1. Several scenarios are possible according to the adiabaticity properties of level crossing near the resonance points. In particular, starting from a pure $\nu_{e L}$ beam at high density, to have a consistent conversion into $\nu_{\mu L}$ at low density the resonance for $\nu_{e L} \rightarrow \nu_{\mu L}^{c}$ has to be crossed non adiabatically, while the passage through the one for $\nu_{e L} \rightarrow \nu_{\mu L}$ has to be adiabatic. However, we have also shown that at very low density, and then in the vacuum, it is more appropriate to deal with the Majorana combinations $\widetilde{n_{ \pm}}$in $(13)$ than with the 
pure flavour states $\nu_{e L}, \nu_{e L}^{c}, \nu_{\mu L}, \nu_{\mu L}^{c}$. This is strictly related to the Dirac-Majorana nature of neutrinos, which chooses Majorana eigenstates instead of pure flavour states as starting points. In this respect, we have to deal with "generic" flavour-changing or flavour-conserving transitions of Dirac-Majorana neutrinos without looking at the particular active neutrino or sterile antineutrino state. It is through the weak interactions, with which neutrinos are produced and detected, that a particular (active or sterile) component of the Majorana eigenstates is chosen.

The results obtained for the case of degenerate Dirac-Majorana mixing are qualitatively valid also in the general case in which all the entries of the Dirac and Majorana mass matrices are non zero and different between them. The main difference between the two cases is that in the general framework there are 3 mass parameters and two mixing angles ruling the evolution, while for the particular case studied in sections 2.3 and 3.4 there are only 2 mass parameters and 1 mixing angle (these parameters being not completely independent, because of relation (50)). The presence of more degrees of freedom in the general case allows to consider some peculiar situations which are not possible otherwise. The most remarkable one is that in the general case the proportionality of the $\Sigma$ parameter to $\sin ^{2} 2 \theta_{+}$(see eq. (50)) is lost, so that the structure of the level crossing diagram at very low density can be altered. The eigenvalues of $H_{m}$ in (62) for zero density (vacuum) are given by

$$
\begin{aligned}
& \frac{1}{8 k}\left( \pm 2 \Delta m_{+}^{2}+\Sigma\right) \\
& \frac{1}{8 k}\left( \pm 2 \Delta m_{-}^{2}-\Sigma\right)
\end{aligned}
$$

so that one can manipulate the mass parameters to modify the low density region of the level crossing diagram without grossly altering the region where the resonance points are present. In any case, there can be present no substantial modifications of the conclusions reached above.

The oscillations of Dirac-Majorana neutrinos here studied with their peculiar features can be efficiently tested in astrophysics, in particular detecting solar or supernova neutrinos, and can have even profound implications in cosmology for the nucleosynthesis of light elements in the Universe.

\section{Acknowledgements}

We express our sincere thanks to Prof. F. Buccella for very useful talks and his unfailing encouragement, and to Prof. E. Kh. Akhmedov for enlightening discussions with one of us (S.E.).

\section{References}

[1] Homestake Collab., Nucl. Phys. B (Proc. Suppl.) 38 (1995) 47;

Kamiokande Collab., Nucl. Phys. B (Proc. Suppl.) 38 (1995) 55; 
Gallex Collab., Phys. Lett., B 327 (1994) 377;

Sage Collab., Phys. Lett., B 328 (1994) 234;

K.Inoue (SuperKamiokande Collab.), talk given at the 5th International Workshop on Topics in Astroparticle and Underground Physics (TAUP 97), Laboratori Nazionali del Gran Sasso, Italy, 7-11 Sept. 1997, to appear in the Proceedings.

[2] J.Bahcall, talk given at 18th Texas Symposium on Relativistic Astrophysics, Chicago 15-20 Dec. 1996, preprint astro-ph/9702057.

[3] P.I.Krastev and S.T.Petcov, Phys. Rev. D 53 (1996) 1665;

S.P.Mikheyev and A.Yu.Smirnov, preprint hep-ph/9708403.

[4] S.Esposito, Mod. Phys. Lett., A 8 (1993) 1557;

N.Hata and P.Langacker, Phys. Rev., D 50 (1994) 632;

Q.Y.Liu and S.T.Petcov, Phys. Rev. D 56 (1997) 7392.

[5] E.Kh.Akhmedov, A.Lanza and S.T.Petcov, Phys. Lett. B 348 (1995) 124;

E.Kh.Akhmedov, talk given at 4th International Solar Neutrino Conference, Heidelberg 8-11 Apr. 1997, preprint hep-ph/9705451.

[6] P.I.Krastev, S.T.Petcov and L.Qiuyu, Phys. Rev. D 54 (1996) 7057.

[7] V.Gribov and B.Pontecorvo, Phys. Lett B 28 (1969) 493;

S.Bilenky and B.Pontecorvo, Phys. Rep. 41 (1978) 225.

[8] L. Wolfenstein, Phys. Rev., D 17 (1978) 2369;

S.P.Mikheyev and A.Yu.Smirnov, Il Nuovo Cimento, 9 C (1986) 17;

Sov. J. Nucl. Phys., 42 (1986) 913; Sov. Phys. Usp., 30 (1987) 759.

[9] E.Kh. Akhmedov, Sov. Phys. JETP 68 (1989) 690.

[10] S.M.Bilenky and S.T.Petcov, Rev. Mod. Phys. 59 (1987) 671.

[11] Y.Fukuda et al., Phys. Lett. B 335 (1994) 237;

R.Becker-Szendy et al., Nuc. Phys. B (Proc. Suppl.) 38 (1995) 331;

W.W.M.Allison et al., Phys. Lett. B 391 (1997) 491;

Y.Totsuka et al., (SuperKamiokande Collaboration), talk given at the 28th International Symposium on Lepton-Photon Interactions, Hamburg 1997, to appear in the Proceedings.

[12] M.C.Gonzalez-Garcia, H.Nunokawa, O.L.G.Peres, T.Stanev and J.W.F.Valle, preprint hep-ph/9801368;

Q.Y.Liu and A.Yu.Smirnov, preprint hep-ph/9712493;

G.L.Fogli, E.Lisi, A.Marrone and D.Montanino, preprint hep-ph/9711421.

[13] R.M.Barnett et al., Phys. Rev. D 54 (1996) 1. 
[14] See, for example, the reviews of F.Boehm, Nucl. Phys. B Proc. Suppl. 48 (1996) 148 and F.Vannucci, ibid. 154.

[15] C.Athanassopoulos et al. Phys. Rev. Lett. 77 (1996) 3082.

[16] M.S.Turner, Talk presented at the International Symposium on Lepton Photon Interactions (IHEP), Beijing, China, 10-15 Aug 1995, preprint astro-ph/9703197; G.Gelmini, Talk given at 5th Mexican Workshop of Particles and Fields, Puebla, Mexico 30 Oct - 5 Nov 1995, prepriut hep-ph/9606409.

[17] A.Kusenko and G.Segre, Phys. Rev. Lett. 77 (1996) 4872.

[18] M.F.Abud, F.Buccella, L.Rosa and A.Sciarrino, Zeit. für Phys. C 44 (1989) 589;

F.Buccella and L.Rosa, Nucl. Phys B (Proc. Suppl.) 28A, (1992) 168;

R.N. Mohapatra and M.K. Parida, Phys. Rev. D 47 (1993) 264.

[19] S.M.Bilenky, C.Giunti and W.Grimus, talk given at 5th International Workshop on Topics in Astroparticle and Underground Physics, Gran Sasso 7-11 Sept. 1997, preprint hep-ph/9711432;

G.L.Fogli, E.Lisi, D.Montanino and G.Scioscia, Phys. Rev. D 56 (1997) 4365;

K.S.Babu, R.K.Schaefer and Q.Shafi, Phys. Rev. D 53 (1996) 606.

[20] T.Yanagida, in "Proc. of Workshop on Unified Theory and Baryon Number of the Universe" (KEK, Japan), 1979,

M.Gell-Mann, P.Ramond and R.Slansky, in Supergravity (eds. P. Van Nieuwenhuizen, D.Z.Freedman),315 (North Holland, 1979).

[21] R.Foot and R.Volkas, Phys. Rev. D 52 (1995) 6595 and ibidem, D 55 (1997) 5147;

Z.G.Berezhiani and R.N.Mohapatra, Phys. Rev. D 52 (1995) 6607.

[22] S.Esposito and N.Tancredi, Mod. Phys. Lett. A 12 (1997) 1829.

[23] S.Esposito, preprint hep-ph/9802336.

[24] B.Pontecorvo, Sov. Phys. JETP VI (1958) 429.

[25] S.Esposito, Il Nuovo Cimento B 111 (1996) 1449.

[26] S.Esposito and G.Capone, Zeit. für Physik C 70 (1996) 55.

[27] T.K. Kuo and J. Pantaleone, Rev. Mod. Phys. 61 (1989) 937. 
Figure 1: Level crossing diagram for $1 \mathrm{MeV}$ momentum Dirac-Majorana neutrinos described by the mass matrices in (46) or (47). The eigenvalues of $H_{m}$ in (87) are plotted versus the density of a medium with $Y_{e}=Z / A=0.48$. Neutrinos parameters are fixed as follows: $\Delta m_{+}^{2}=10^{-6} \mathrm{eV}^{2}, \Sigma=2.5 \times 10^{-7} \mathrm{eV}^{2}$, sin $2 \theta_{+}=0.1$. Dashed lines refer to the zero mixing limit $\left(\theta_{+}=0\right)$ of the energy eigenvalues. 


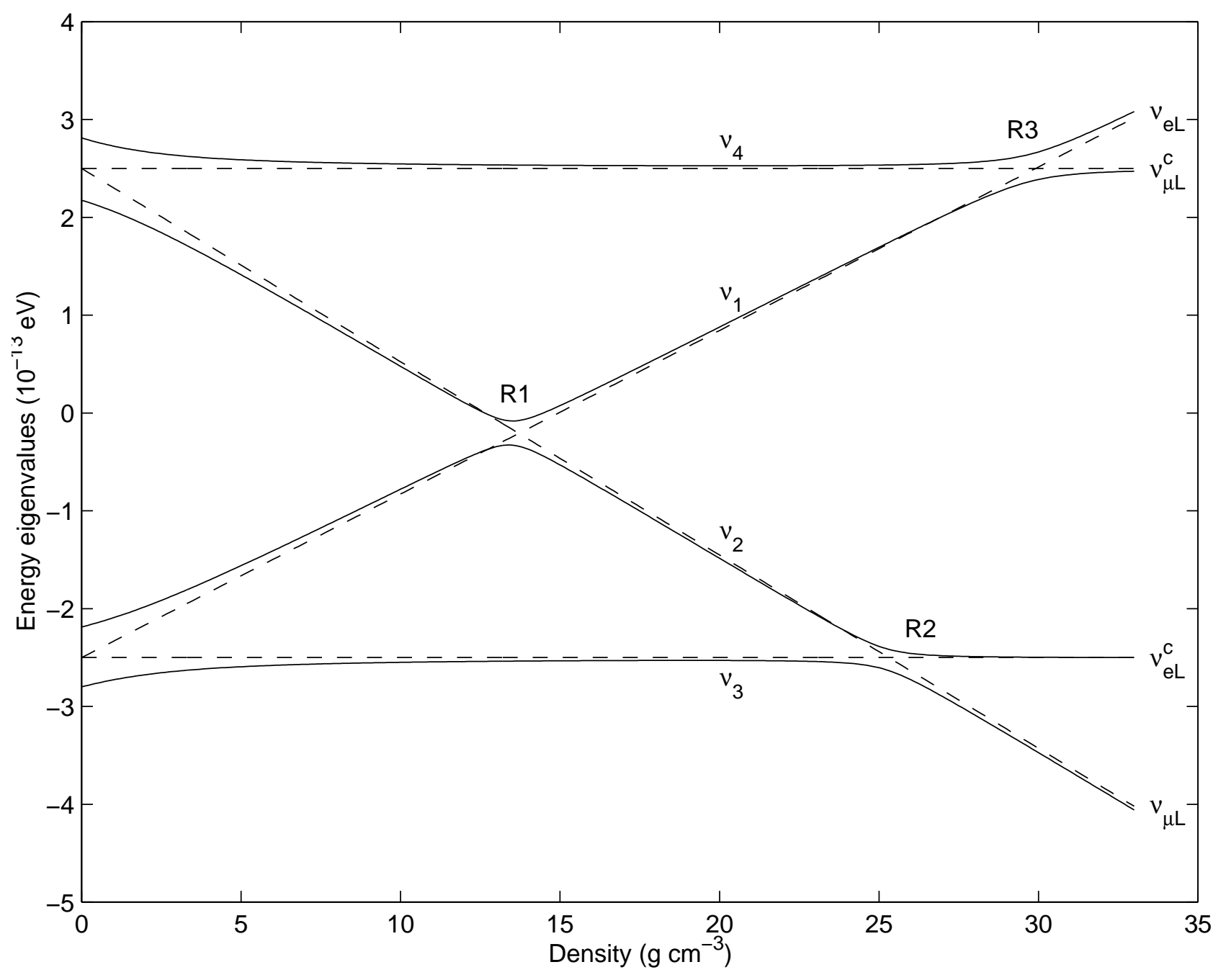

